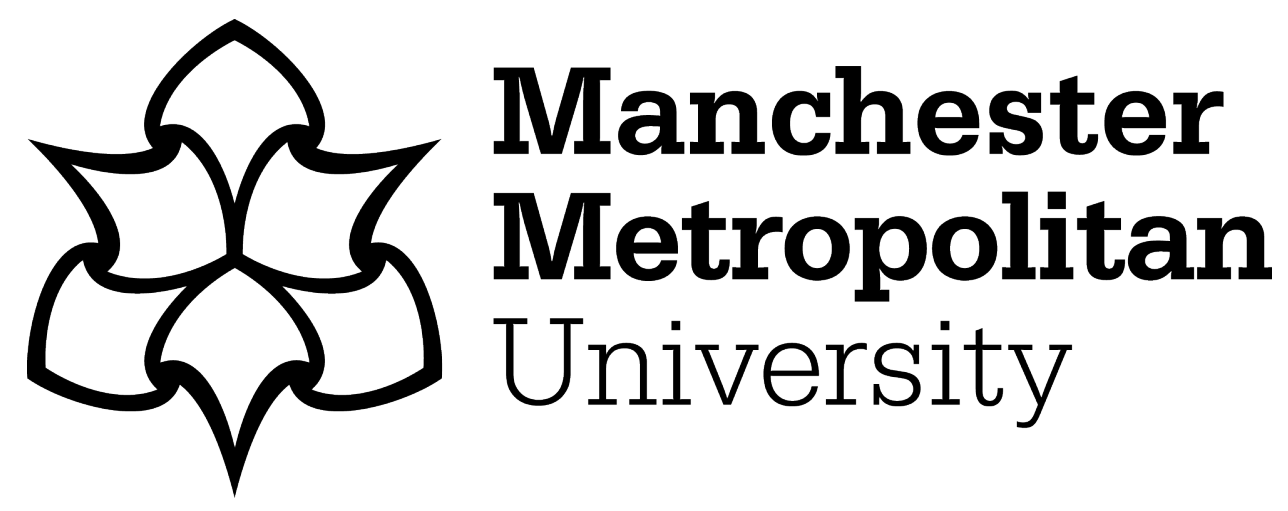

Ashraf, I, Zikria, YB, Hur, S, Bashir, AK, Alhussain, T and Park, Y (2021) Localizing pedestrians in indoor environments using magnetic field data with term frequency paradigm and deep neural networks. International Journal of Machine Learning and Cybernetics, 12 (11). pp. 3203-3219. ISSN 18688071

Downloaded from: https://e-space.mmu.ac.uk/627677/

Version: Accepted Version

Publisher: Springer Verlag

DOI: https://doi.org/10.1007/s13042-021-01279-8

Please cite the published version 


\title{
Localizing Pedestrians in Indoor Environments Using Magnetic Field Data With Term Frequency Paradigm and Deep Neural Networks
}

\author{
Imran Ashraf $^{\dagger}$. Yousaf Bin Zikria ${ }^{\dagger}$. Soojung Hur • Ali Kashif Bashir . \\ Thamer Alhussain • Yongwan Park*
}

Received: date / Accepted: date

\begin{abstract}
Indoor environments are challenging for global navigation satellite systems and cripple its performance. Magnetic field data-based positioning and localization has emerged as a potential solution for ubiquitous indoor positioning and localization. The availability of embedded magnetic sensors in the smartphone simplifies the positioning without the additional cost of infrastructure. However, the data divergence due to smartphone heterogeneity circumscribes the wide applicability of magnetic field-based positioning approaches. This research proposes the use of term frequency (TF) extracted from the magnetic field data to alleviate the impact of smartphone heterogeneity. For this purpose, the magnetic field data are transformed into terms (words) and documents. Extracted TF vectors are used to train long short term memory and gated recurrent unit networks. A voting scheme is contrived to incorporate the predictions from these networks. Experiment results with three different smartphones like LG G6, Galaxy S8,
\end{abstract}

Imran Ashraf, Yousab Bin Zikria, Soojung Hur, and Yongwan Park

Department of Information and Communication Engineering, Yeungnam University, Gyeongsan 38541, South Korea

E-mail: imranashraf@ynu.ac.kr, yousafbinzikria@ynu.ac.kr, sjheo@ynu.ac.kr

Ali Kashif Bashir

Department of Computing and Mathematics, Manchester Metropolitan University, Manchester, United Kingdom

E-mail: dr.alikashif.b@ieee.org

Thamer Alhussain

E-commerce Department, College of Administrative and Financial Sciences, Saudi Electronic University Riyadh, Saudi Arabia

E-mail: talhussain@seu.edu.sa

* Corresponding author, Yongwan Park

E-mail: ywpark@yu.ac.kr

$\dagger$ Primary first authors, equal contributions from these authors. and LG Q6 demonstrate that the use of TF mitigates the impact of the smartphones' variability. Performance comparison with state-of-the-art approaches reveals that the proposed approach performs better than those of other approaches in alleviating the influence of using various smartphones for magnetic field-based indoor localization. Furthermore, the localization performance of the proposed is better than those of other approaches, even using a smaller amount of magnetic field data.

Keywords Indoor positioning and localization . magnetic field data $\cdot$ term frequency $\cdot$ deep neural networks $\cdot$ smartphone sensors

\section{Introduction}

Positioning and localization became the talk of the town during recent years. Location-based services (LBS) accelerated the pace of research and development for both outdoor and indoor positioning and localization technologies. The global navigation satellite system (GNSS) is a de-facto technology that serves the outdoor position with a high accuracy [1]. Still, its performance is crippled in many challenging environments where the user is walking through canyons, congested areas with tall buildings, and indoor environments especially [2]. Consequently, for such and other similar complicated environments, a large variety of positioning and localization technologies has been put forward under the banner of infrastructure-based and infrastructure-less approaches. The approaches in the former category rely upon the custom infrastructure like beacons, sensors, and tags, etc. to sense the environment and perform positioning. On the other hand, the approaches that belong to the latter category leverage the opportunistic signals present in the environment. Radiofrequency 
identification (RFID), ultra-wideband (UWB), and Bluetooth (BLE), etc. are part of the former category while pedestrian dead reckoning (PDR) and magnetic fieldbased positioning (MFP) approaches are examples of the latter category [3-5]. Although Wi-Fi positioning requires Wi-Fi access points (APs), yet it is put in the latter category due to the wide installation of Wi-Fi APs at public places and indoor environments [6].

The Wi-Fi positioning has been investigated extensively for two decades and provides an average accuracy of 5 to $6 \mathrm{~m}$ [7]. Despite the wide prevalence of Wi-Fi APs, simplicity, and provided accuracy, Wi-Fi approaches are not suitable to meet the standards of indoor localization due to many reasons. Predominantly, Wi-Fi positioning utilizes the received signal strength indicator (RSSI) of present APs to locate a user and suffer severe performance degradation while positioning in dynamic environments. Radio signal propagation is affected by human mobility that leads to unaccounted RSSI variability thus affecting the localization accuracy. Shadowing, signal blockage, and multipath are only a few among many problems that Wi-Fibased approaches face. In addition to that, time, user and device variability affect the performance of such systems as well [8]. Apart from that the introduction of 'throttle' in Android 9.0 restricting frequent scanning of Wi-Fi APs limits the wide use of Wi-Fi positioning and localization approaches. BLE has been the focus of interest for indoor positioning technology due to its high accuracy. However, BLE set up requires the dense deployment of BLE beacons to provide high accuracy. Since BLE is based on radio signal propagation, it has inherent limitations of radio signal propagation including shadowing, signal absorption, and multipath. Its performance is easily affected due to human mobility.

PDR provides only a relative position and always needs a starting/previous position to infer the next position. So, it works as a complementary approach to improve the localization accuracy.

Magnetic field-based indoor positioning and localization has emerged as a prospective candidate for indoor positioning. The ubiquity of the magnetic field makes it an attractive and influential solution for indoor localization. The wide proliferation of smartphones with embedded magnetic sensor offers the opportunity to use magnetic field data for positioning and localization without additional sensors and infrastructure. As a consequence, many indoor positioning and localization works have been presented that focus on the use of the magnetic field data $[9,10]$. One limitation that undermines the wide use of such approaches is smartphone heterogeneity. Today, a wide range of smart- phones is manufactured by many companies like Samsung, LG, Apple, Huawei, and Nokia, etc. These smartphones carry magnetic sensors from different vendors having various specifications. The magnetometer in various smartphone models from the same company is not alike either as they have different sensitivity and noise tolerance. As a result, the collected magnetic field data from smartphones vary significantly and localization accuracy largely fluctuates even with the same localization approach. It seems very difficult to devise an approach that can work seamlessly with various smartphones and provide a similar localization accuracy. This study proposes the use of term frequency (TF) to overpower this issue and mitigate the impact of device heterogeneity on localization. The contributions of this study are summarized as follows

- An indoor localization approach is devised that leverages the magnetic field data from the embedded magnetic sensor of the smartphone. The data are normalized using a modified min-max normalization approach to mitigate data variability caused by heterogeneous devices.

- The term frequency paradigm is proposed to alleviate the impact of device heterogeneity. The process of transforming the magnetic field data to terms and documents is presented.

- Deep neural networks like long short term memory and gated recurrent unit networks are structured that utilize the extracted TF for training and testing. A voting criterion is improvised to incorporate the predictions from neural networks to estimate the user's current location.

- Three smartphones including LG G6, Galaxy S8, and LG Q6 are used to carry out the experiments to analyze the impact of smartphone heterogeneity on the proposed approach.

- Results are compared with three state-of-the-art indoor localization approaches that utilize the magnetic field data for localization. Performance analysis reveals that the proposed approach performs better than those of state-of-the-art approaches.

The rest of the paper is organized in the following manner. Section 2 discusses research works related to the current study. Section 3 gives an overview of the challenges to use the magnetic field data for indoor localization. The working of the proposed approach for transforming the magnetic field data into terms, extraction of TF for a document, and localization algorithm are discussed in Section 4. Results are discussed in Section 8 while the conclusion is given in Section 6 . 


\section{Related Work}

The vast majority of the magnetic field based indoor localization works use the fingerprinting approach for its ease of adaption, simplicity, and effectiveness. Fingerprinting involves offline and online phases where the former refers to collecting the data on ground truth points to make the database while the latter phase includes the localization using the smartphone data from the user smartphone at run time. The ground truth points are the designated locations with known coordinates and are traditionally separated by equal distances. Concerning the used technology for localization, MFP can be divided into groups: approaches based on magnetic field data alone, hybrid approaches that utilize complementary technologies like BLE, Wi-Fi, and PDR, etc. to improve localization performance.

Authors leverage the magnetic field data to identify various rooms in a building [11]. For this purpose, the database is made using the fast Fourier transform (FFT) of the magnetic field data as the signature. Acer A500 tablet is used worn around the waist and the data is collected at $100 \mathrm{~Hz}$ for 10 seconds. The user's position is estimated by comparing the FFT of the collected signal with that of the fingerprint database using a modified Manhattan distance. The proposed approach identifies all the rooms correctly, however, the precise position of the user is not evaluated. The user's exact position inside the room cannot be estimated correctly as the approach works on the room level only. In addition to that user has to move from one specific point to another to identify a specific floor that is restricting and limiting the scope of indoor positioning [12].

Indoor infrastructures comprise locations with particular signatures that are influential than those of other locations. Such unique locations are often called, "landmarks" and can dramatically improve the localization performance of MFP approaches. For example, the approach presented in [13] utilizes magnetic landmarks to perform indoor localization. Landmarks are selected based on the minima/maxima in the magnetic field data considering eight connected neighbors. Deep neural networks are trained using features of 'recurrence plot', 'trend' of the peaks, and peak-to-peak 'length' from the magnetic data. The trained DNN can correctly classify $80 \%$ of the magnetic landmarks. However, the meter level or the exact location of the user is not calculated with the proposed approach. Furthermore, the magnetic landmarks can be several meters apart which increases location uncertainty. In addition to that, the magnetic field data from seven meters long place is gathered to identify a landmark that is long and might not be appropriate or available in many cases.
Hybrid approaches offer higher accuracy than those of using the magnetic field data alone and present a more practical and effective solution. As a result, the data from multifarious sensors like $\mathrm{Wi}-\mathrm{Fi}$, accelerometer, BLE, etc., is used to enhance the accuracy of MFP. For example, a sensor fusion approach is presented in [14] that maneuvers the data from WiFi, smartphone camera, magnetometer, Bluetooth, and people's co-occurrence. Initially, camera images are used to identify the coarse location of the user which is used to narrow down the search space for the magnetic field database. On the other hand, Wi-Fi works to correct the location estimation periodically. It helps to both elevating the accuracy and reducing battery consumption. Results show that the user can be located correctly with an accuracy of $83.7 \%$ with the proposed approach. Moreover, if the user time-specific activities are considered, the location accuracy can be further improved. The use of BLE proves to enhance the accuracy in multi-sensor indoor positioning approaches, where multiple scenarios can be adopted. Two kinds of approaches are adopted for BLE based indoor positioning such as hybrid solutions as in $[15,16]$ or dense deployment of BLE beacons [17]. Besides the additional cost for deploying the beacons, BLE has other issues. It is based on radio propagation which has its inherent limitations. These limitations are prevalent both in time-based positioning approaches like multilateration and RSSI based approaches. Such problems include multipath, scattering, shadowing, and signal absorption due to human mobility. Using the sparse deployment of BLE beacons is useful to estimate course location which can be used to narrow the search space in hybrid solutions to reduce the processing time and increase the positioning accuracy

The use of deep learning approaches also helps to improve the localization performance with the magnetic field data. In this regard, authors in [18] present the use of convolutional neural networks to identify the indoor scene that functions as an important module in an indoor localization system. Scene recognition lowers the search space of the magnetic field database and improves time and performance. Motion sensors, compass, and Wi-Fi further increase the localization accuracy. Sensor fusion using particle filter is adopted to this end. The reported localization accuracy of the system is $1.32 \mathrm{~m}$ at $95 \%$.

The above-cited research works focus on using a single smartphone primarily and do not investigate the impact of device heterogeneity on the presented localization approach. Authors make a profound study of how walking speed and smartphone disparity affect the magnetic field data in [19]. They present an indoor po- 
sitioning and tracking approach that incorporate Wi-Fi signals, PDR, and magnetic field data to track the user. The fusion of Wi-Fi helps in reducing the error dramatically. Mean, 2nd and 3rd quartile errors are low, however, the localization performance with multiple smartphones is not tested.

Several works emphasize using multiple smartphones to analyze the impact of device heterogeneity with magnetic field data. By way of example, authors present a sensor fusion approach in [20] for magnetic field-based indoor localization with multiple smartphones. Contrary to the traditional approach of using the intensity, the patterns formed by the magnetic field data are used to mitigate the influence of device heterogeneity. Data from motion sensors including an accelerometer and the gyroscope helps in reducing large errors and improves the localization accuracy. Experimental results indicate that the use of magnetic field data patterns reduces the effect of using diversified smartphones. Similarly, an indoor localization approach is presented in [9] that investigates how multifarious smartphone can compromise the localization accuracy when used with the magnetic field data. Two smartphones like Samsung Galaxy S8 and LG G6 are tested on the fingerprinting approach where the database is built using S8 while the testing is done with both the smartphones. A modified particle filter approach is used for improving the localization accuracy with multiple sensors. Results show that the proposed approach can alleviate the impact of using various devices.

In the same fashion, authors use different smartphones to perform indoor localization with a magnetic field fingerprint database in [21]. Dynamic time warping (DTW) is used for matching the user collected magnetic field data sample to the stored magnetic sample and 35 magnetic samples are taken in this regard. The classification accuracy is $88 \%$ where accuracy refers to the number of correctly predicted positions concerning total predictions. A total of 16 test signatures are used to evaluate the accuracy of the proposed approach. The localization error falls between 0 to $17 \mathrm{~m}$ and varies in different buildings as well as for different lengths of test signatures. Another work in the same line is [22] that takes advantage of the deep learning approach to overcome the limitations of device heterogeneity. Multiple neural networks are trained on the patterns of the magnetic field data to estimate the user's location. Although the localization results are promising, yet, the device dependence is not resolved fully. The abovediscussed research works investigate the use of multiple smartphones from various companies to analyze how changing the localization device impacts the localization accuracy. Results demonstrate the reduction in de- vice dependency, yet, the proposed approaches are not device-independent. Localization accuracy though similar, yet, the difference is rather substantial. So, the current study aims to alleviate device dependency to make the localization performance of various smartphones substantially similar.

Table 1: Summary of the research works discussed in related work.

\begin{tabular}{|c|c|c|c|}
\hline Ref. & Approach & Accuracy & Shortcoming \\
\hline$[9]$ & $\begin{array}{l}\text { Multi-sensor } \\
\text { fusion }\end{array}$ & Meter level & $\begin{array}{l}\text { Fixed orientation of } \\
\text { the smartphone. }\end{array}$ \\
\hline$[11]$ & $\mathrm{FFT}$ & Room level & $\begin{array}{l}\text { User moves between } \\
\text { specific points }\end{array}$ \\
\hline [13] & $\begin{array}{l}\text { Magnetic } \\
\text { landmarks }\end{array}$ & Landmarks & $\begin{array}{l}\text { Meter level accuracy } \\
\text { is not provided }\end{array}$ \\
\hline [14] & $\begin{array}{l}\text { Multi-sensor } \\
\text { fusion }\end{array}$ & Meter level & $\begin{array}{l}\text { User activity tracking } \\
\text { has privacy issues }\end{array}$ \\
\hline$[16]$ & $\begin{array}{l}\text { Hybrid solu- } \\
\text { tion }\end{array}$ & Meter level & $\begin{array}{l}\text { High infrastructure } \\
\text { dependence. }\end{array}$ \\
\hline$[17]$ & BLE & Meter level & $\begin{array}{l}\text { Dense deployment of } \\
\text { BLE beacons is re- } \\
\text { quired. }\end{array}$ \\
\hline [18] & $\begin{array}{l}\text { Multi-sensor } \\
\text { fusion }\end{array}$ & Meter level & $\begin{array}{l}\text { High computational } \\
\text { cost and time. }\end{array}$ \\
\hline [19] & $\begin{array}{l}\text { Wi-Fi \& } \\
\text { smartphone } \\
\text { sensors }\end{array}$ & Meter level & $\begin{array}{l}\text { Infrastructure and } \\
\text { device dependence. }\end{array}$ \\
\hline$[20]$ & $\begin{array}{l}\text { Multi-sensor } \\
\text { fusion }\end{array}$ & Meter level & $\begin{array}{l}\text { Longer magnetic field } \\
\text { data is required. }\end{array}$ \\
\hline$[21]$ & $\begin{array}{l}\text { Multi-sensor } \\
\text { fusion }\end{array}$ & Meter level & $\begin{array}{l}\text { Very long magnetic } \\
\text { field data is required. }\end{array}$ \\
\hline$[23]$ & $\begin{array}{l}\text { Multi-sensor } \\
\text { fusion }\end{array}$ & Meter level & $\begin{array}{l}\text { High computation } \\
\text { complexity, device } \\
\text { dependency. }\end{array}$ \\
\hline
\end{tabular}

An important work, similar to ours, is [23] that introduces the use of a bag of words (BoW) approach for indoor localization with the magnetic field data. Each location serves as a different class having BoW features. The research aims to solve the speed invariance problem for magnetic field-based indoor localization where the different speeds of the users affect the localization accuracy. Several classifiers are used to predict the class using the extracted BoW. The scope of this research is however restricted by two major limitations. First, experiments are conducted using a single smartphone and smartphone heterogeneity is not studied at all. Second, the evaluation is based on the classification accuracy which does not show mean, median, 50\%, 75\%, and maximum errors that are key indicators to prove the performance of positioning and localization. Thirdly, very long sequences of few tens of seconds of magnetic field data are used to make a reference point. The use of long sequences of data is not appropriate, not to men- 
tion the fact that many complicated indoor areas do not have much longer sequences at all. Table 1 summarizes the discussed research papers in the related work.

The current study introduces the TF paradigm to perform indoor localization using deep neural networks. Various algorithms are proposed to transform the magnetic field data into terms and documents, as well as, incorporating the predictions of neural networks to estimate the user's current location. Device heterogeneity is extensively studied using three different smartphones in this regard.

\section{Challenges of using Magnetic Field Data for Localization}

The earth's magnetic field referred to as the 'magnetic field' here for simplicity is a natural phenomenon caused by the flow of convection current. Its distribution over the globe is uniform with a smooth change between $20 \mu \mathrm{T}$ to $65 \mu \mathrm{T}$. At any given point, it has $x, y$, and $z$ values that represent the magnitude of the magnetic field in the North, East, and downward direction. These values do not change abruptly and remain similar in the absence of man-made constructions. However, the buildings containing ferromagnetic materials like nickel, cobalt, and iron, etc. interfere with the natural phenomenon and cause disturbances, often called, 'anomalies'. Such disturbances are observed to present unique values at different places and used as 'signature' to make fingerprinting databases [24].

There are two well-known representations to show the magnetic field at a given point. One way is to use magnetic $x, y$, and $z$ for North, East, and downward components, respectively. An alternative approach used in a few research works is through $F, I$, and $D$ that corresponds to total magnetic field intensity, inclination, and declination, respectively [25]. The latter representation is not very famous with positioning approaches concerning the sensitivity of the components. The $I$, and $D$ are measured in angles using the horizontal component $H=\sqrt{x^{2}+y^{2}}$ and magnetic $x, y$, and $z$. Small variations in these components can easily change the values for $I$, and $D$ which ultimately affects the positioning accuracy. Predominantly, magnetic $x, y$, and $z$ are used to formulate the fingerprinting database for MFP systems. The major drawback of using $x, y$, and $z$ is their variability concerning the smartphone used. For example, Figure 1 shows the magnetic $x, y$, and $z$ collected using three different smartphones walking along with the same location at the same time. Despite that, the values of these components are not the same and vary significantly.

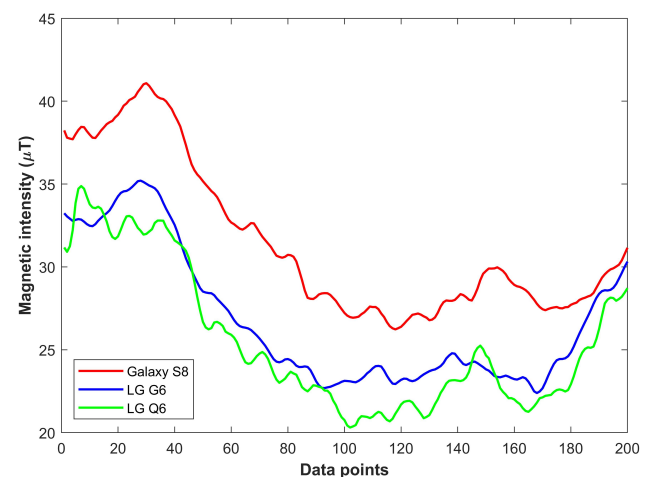

(a)

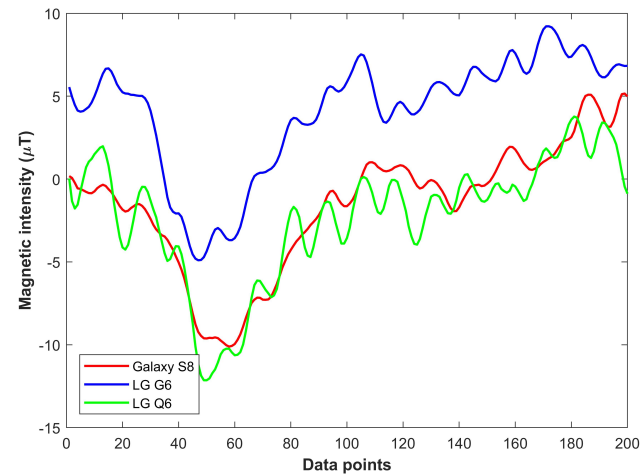

(b)

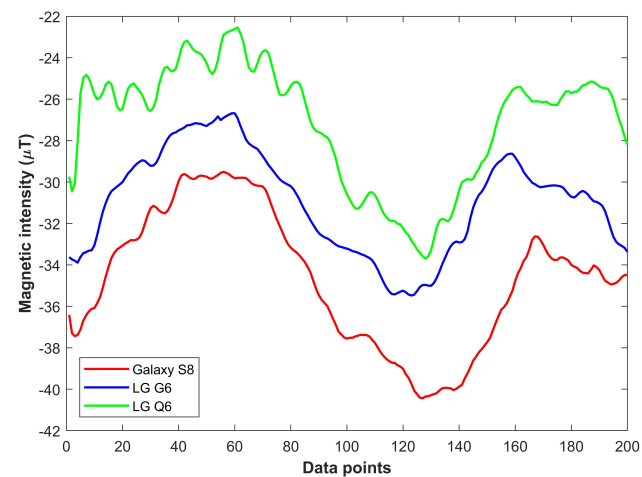

(c)

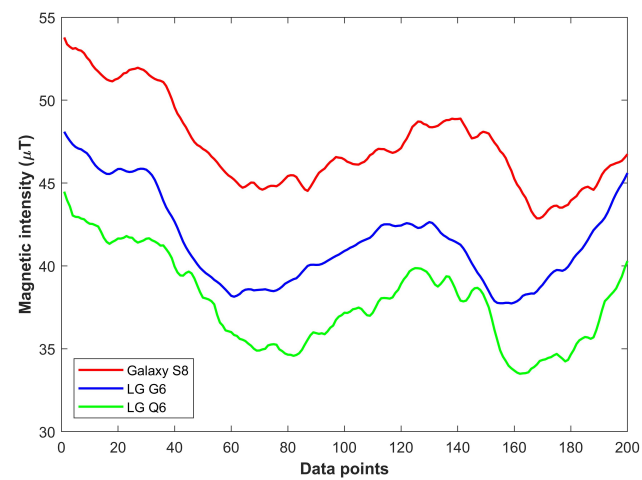

(d)

Fig. 1: Magnetic field data collected using heterogeneous smartphones for the same location, (a) magnetic $x$, (b) magnetic $y,(\mathbf{c})$ magnetic $z,(\mathbf{d})$ magnetic $F$ 
The magnetic field data shown in Figure 1 indicates that the intensity is not the same with different devices. Consequently, when the localization approach is used on the data from different devices, the localization accuracy varies significantly. So, devising an approach that can work seamlessly and provide similar accuracy with heterogeneous devices is very important.

\section{Materials and Methods}

This section contains the information on the term frequency paradigm, how the magnetic field data is transformed into terms and documents, as well as, the localization algorithms.

\subsection{Term Frequency Paradigm}

Training the neural networks requires feature vectors from the magnetic data. So, the magnetic field numeric data needs to be transformed feature vectors. Several techniques like BoW, GLoVe, Word2Vec, and TF, etc. can be used for this purpose. The accuracy of BoW is limited by character-length limitations and insufficient word occurrences [26]. Additionally, it is often difficult and computationally expensive to build a vocabulary large enough to cover all the possible words [27]. GloVe (Global Vectors) for word representations is another approach used to create word embedding. The GLoVe works like matrix factorization and is widely used in text mining tasks. It has advantages when used with a large corpus concerning its scaling capability, yet, often not efficient with smaller data [28]. Word2Vec is an approach designed by Google for text classification and it uses CBOW (Continuous BOW). Although showing promising results with English text classification, it makes context-based predictions and it not appropriate for the current task [29]. TF is one of the most widely used approaches in text mining that shows outperforming results in natural language processing, sentiment analysis, and text classification [26]. We aim to utilized TF concerning to take advantage of its superior performance. It counts the words in a document and produces a matrix that represents the total number of occurrence (frequency) of each word in a given document. Frequency is adjusted by the length of a document. Mathematically, TF for the word $t$ in the document $d$ from $D$ document set can be calculated as

$$
t f(t, d)=\log (1+f(t, d))
$$

where $f$ represents the frequency of the term $t$ in the document $d$.
Equation 1 calculates the frequency of each term. In other words, it counts, how many times a specific term has appeared in a given document. The TF feature counts the frequency of the terms in the document and does not measure the commonality or rarity of the term. Take, for example, the following statements:

"Brevity is the soul of wit",

"Beauty comes from the soul",

"Perception of beauty is different than beauty itself".

Table 2: TF feature matrix for the above-given statements.

\begin{tabular}{l|lll}
\hline Term & \multicolumn{3}{|c}{ Term frequency } \\
\hline brevity & 0.6931 & 0.0000 & 0.0000 \\
soul & 0.6931 & 0.6931 & 0.0000 \\
wit & 0.6931 & 0.6931 & 0.0000 \\
beauty & 0.0000 & 0.6931 & 1.0986 \\
come & 0.0000 & 0.0000 & 0.0000 \\
perception & 0.0000 & 0.0000 & 0.6931 \\
different & 0.0000 & 0.0000 & 0.6931 \\
\hline
\end{tabular}

The TF vector for the above-given statements is given in Table 2. The TF matrix of the document $d$ is used to train and test classification algorithms to classify the given documents. TF is amongst the widely used techniques for text document classification. However, we utilize it to solve the problem of magnetic field-based indoor localization. For this purpose, we first need to define terms and documents regarding localization.

4.2 Transforming the Magnetic Field Data into Terms and Documents

In the text analysis scheme, a document is comprised of a sequence of various terms (words) where each term is an ordered sequence of characters. Various documents contain various terms as well as, the order of the terms may be different. Text analysis focuses on measuring the similarity of these terms to determine the similarity of the documents. By way of the same analogy, the term $t$ in the localization scenario is a pattern formed using the magnetic field data and each data value resembles a character $c$. The sequence of these patterns constitutes a document $d$ using the magnetic field data. The document $d$ represents a specific location $L$ that corresponds to $(x, y)$ coordinate. Vocabulary $V$ refers to the collection of all unique terms $T$ and is used as a reference to look up the terms during the localization process. Table 3 show the list and explanation of the notations used in Algorithms 1 and 2. 
Table 3: Notations used in Algorithm 1 and Algorithm 2 .

\begin{tabular}{ll}
\hline Notation & Description \\
\hline$T$ & Term indicating a unique pattern of data. \\
$D$ & document that refers to location. \\
$L$ & Location in 2D, i.e., $(x, y)$ \\
$V$ & Vocabulary, collection of all terms (patterns). \\
$M$ & Magnetic field data. \\
$x$ & North component of the magnetic field. \\
$y$ & East component of the magnetic field. \\
$z$ & Downward component of the magnetic field. \\
$M_{d}$ & Denoised magnetic field data. \\
$\widehat{M}$ & Normalized magnetic field data. \\
$d i s t$ & Euclidean distance between two positions. \\
$l s t m_{p}$ & Predictions from LSTM. \\
$g r u_{p}$ & Predictions from GRU. \\
$P$ & Set of unique predictions from LSTM and GRU. \\
$O$ & Occurrences of each unique position. \\
$L_{c}$ & Candidates for probable location of the user. \\
$L_{u}$ & Estimated current location of the user. \\
\hline
\end{tabular}

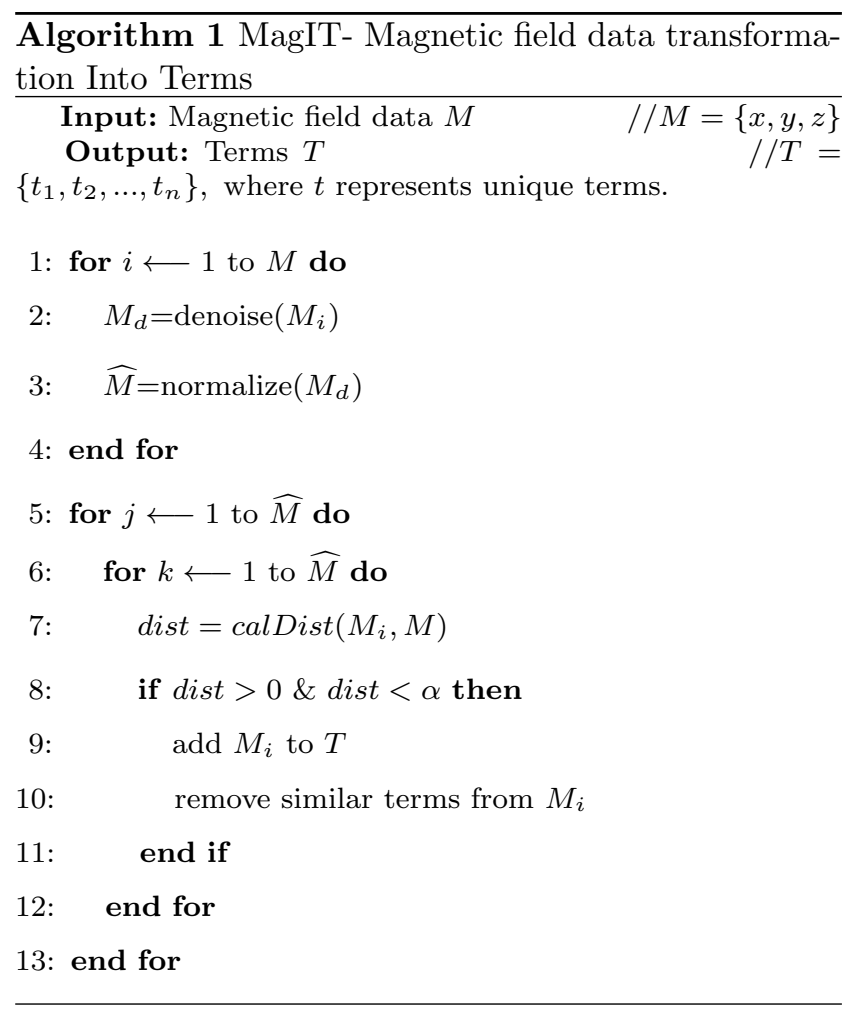

Let $M$ be the magnetic field data where $M=\{x, y, z, F\}$ and each of $x, y, z$, and $F$ is $\{1,2, \ldots, n\}$ with $n$ as the total number of data samples, the term $t$ can be formed as

$$
t=M_{i 1}, M_{i 2}, \ldots, M_{k}
$$

So, the term $t$ is an ordered sequence of $k$ elements from each of magnetic $x, y, z$, and $F$. It shows the sequence of the magnetic field data in the order of collection and refers to the data collected from the embedded magnetic field sensor of the smartphone. The document $d$ here refers to the collection of these terms at a reference location in the indoor location. Hypothetically, each document contains a combination of different terms, so it is possible to discriminate one document (location) from the other. However, to extract TF for a given document, first, we need to identify all possible terms given in document set $D$ where $D$ refers to the indoor location where the localization is to be performed. Algorithm 1 is designed to carry out this operation.

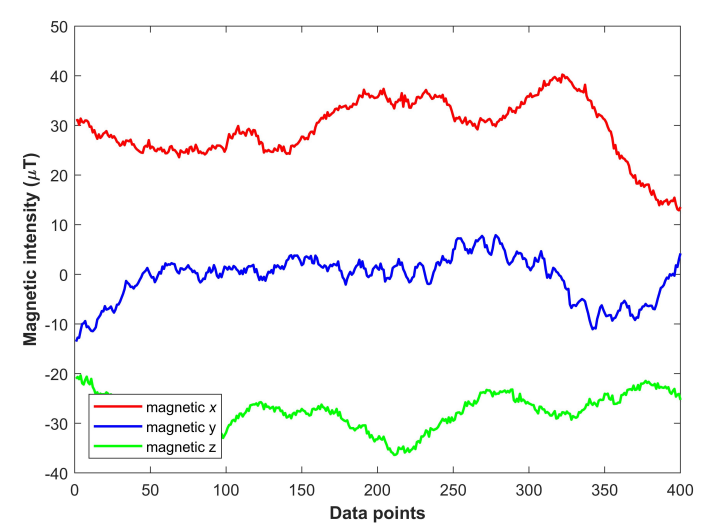

(a)

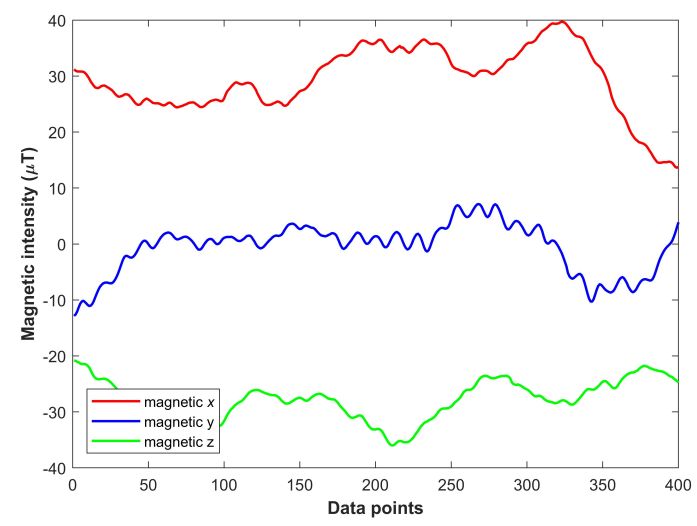

(b)

Fig. 2: The denoising of the magnetic field data using wavelet denoising, (a) before denosing, (b) after denoising.

Lines 1-4 perform two operations on the input magnetic field data $M$; denoising and normalization. The magnetic data collected from the magnetic sensor of the smartphone contains noise due to the sensitivity of the sensor and slight movements of the user's hands and has to be removed for better performance of MFP systems. Wavelet denoising is utilized in this regard. 
Figure 2 shows the magnetic field data before and after denoising.

Once the data $M$ are denoised into $M_{d}$, it is normalized using a modified min-max normalization method using

$$
\widehat{M}=\frac{M_{d_{i}}-\min \left(M_{d_{n}}\right)}{\max \left(M_{d_{n}}\right)-\min \left(M_{d_{n}}\right)}
$$

where $i$ represents $i$ th sample in $M_{d}$, min, and max are the minimum and maximum samples while $n$ represents the total number of samples considered for normalization. In other words, $n$ represents the number of samples that are considered to define a term. Unlike text processing, where each term has a different length, we consider equal length for all the terms. The difference lies, not in their length but their shape/pattern.
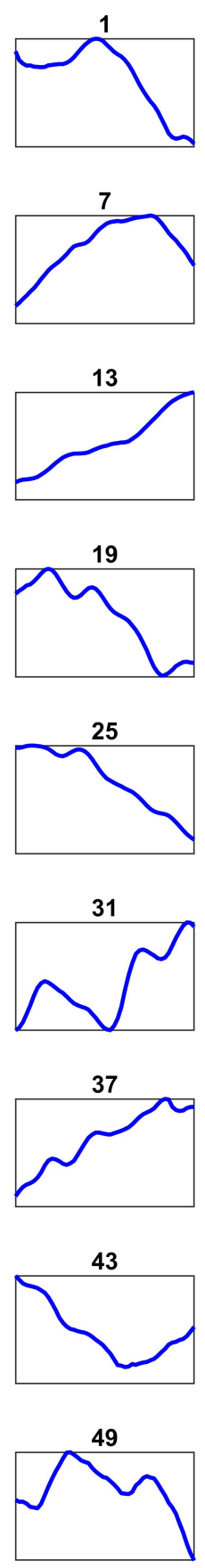
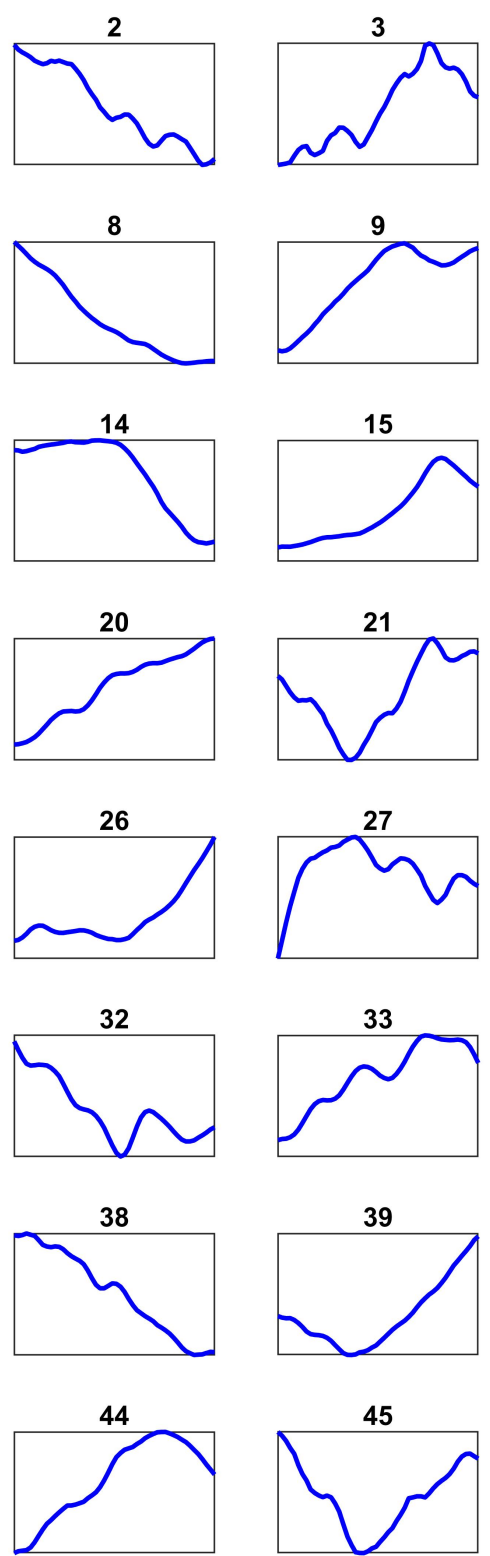

15
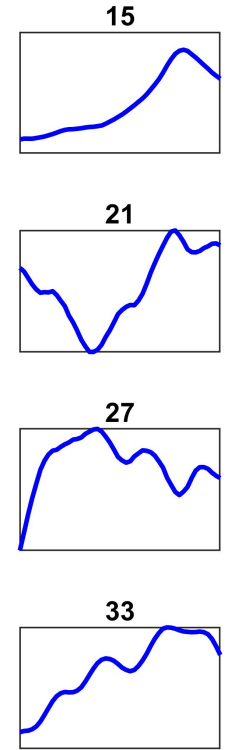

39

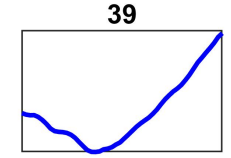

45

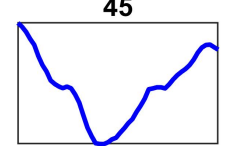

50
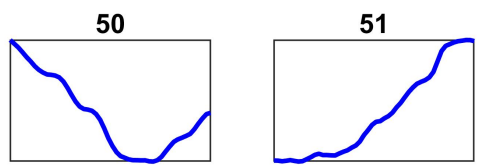
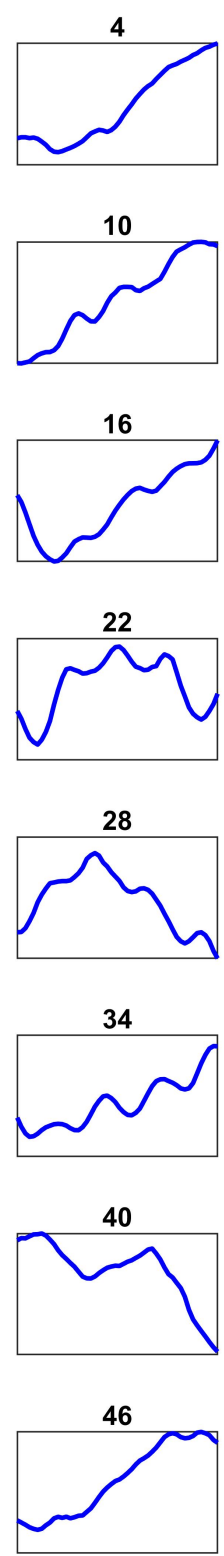
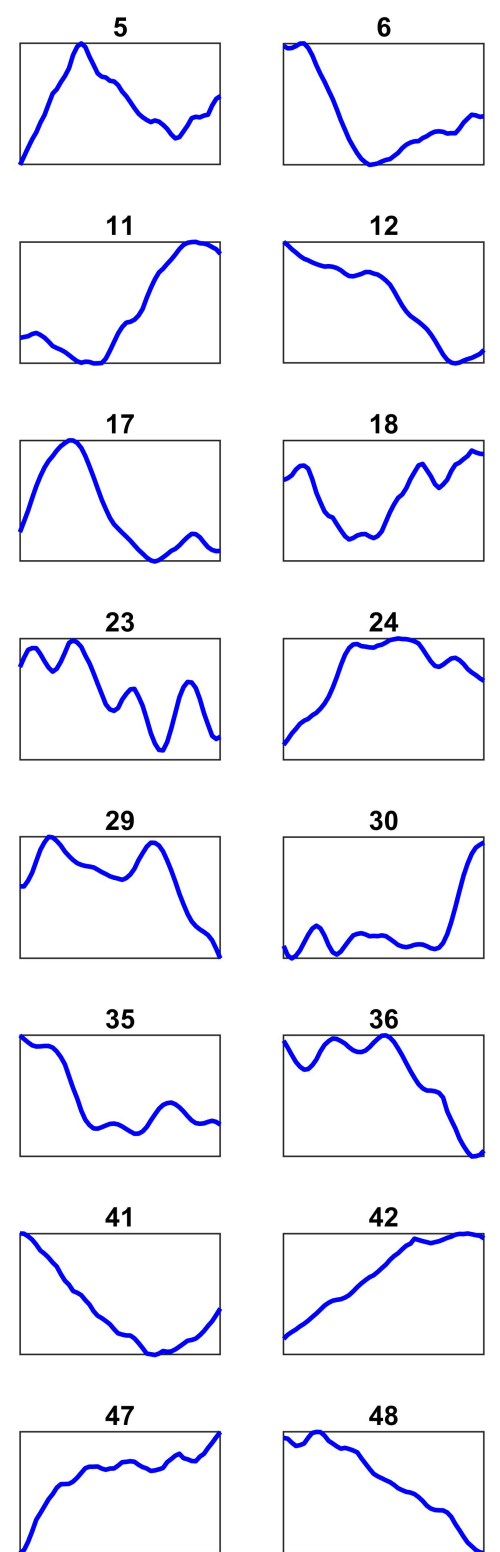

30
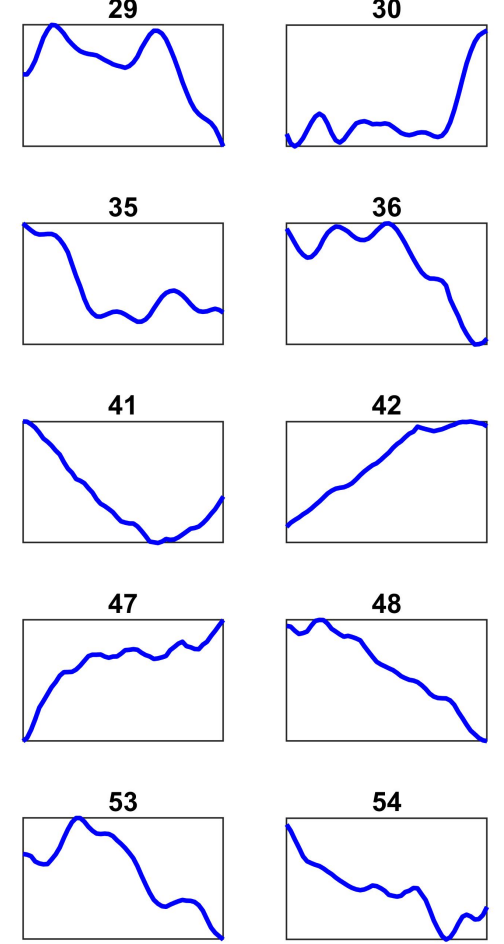

Fig. 3: Examples of terms found from the magnetic field data after normalization. 
The patterns formed by the magnetic field data are almost similar, however, the matching of these patterns is not a trivial problem and requires substantial computational resources concerning image-based matching. The normalization process is carried out to put these patterns on the same scale so that the pattern matching can be done without image-based matching techniques.

Figure 3 shows a few terms from the magnetic field data after lines 1-4 of Algorithm 1 are executed. The terms displayed in Figure 3 are not unique, for example, terms number 2 and 38 look very similar, if not the same. Figure 4 shows a few terms in each graph with a high resemblance. Similar to text analysis, where each term has a unique sequence of characters, we need to select terms that represent unique patterns formed by the magnetic field data.

Lines 6-13 contains the procedure to select only one term from those with high similarity. For this purpose, a single term is taken at a time and its Euclidean distance with all other terms is calculated using

dist $=\sqrt{\left(M_{i}-M_{k}\right)^{2}}$

where $M_{i}$ is the term considered to check its similarity with $k$ elements of $\widehat{M}$. Equation 4 gives the quantifiable difference between two given magnetic field sequences.

Selection of unique terms and deletion of duplicate terms is based on the criteria

$$
\left\{\begin{array}{lr}
\text { dist }>0 \text { \& dist }<\alpha & \text { similar term } \\
\text { else } & \text { unique term }
\end{array}\right.
$$

where $\alpha$ is the threshold set to decide the similarity of a term. Equation 5 is used to discriminate two sequences of the magnetic field data. It distinguishes one magnetic field sequence from others using $\alpha$ which is an empirical value and its value is set to 0.4 . The value is determined through experiments where the impact of various values is analyzed concerning the dissimilarity between given terms. The set value is a trade-off between the size of the vocabulary (processing time) and accuracy for positioning. Increasing its value leads to a reduced number of unique terms which lowers the positioning performance while decreasing its value gives a higher number of unique terms, elevates the positioning accuracy, and increases the processing time. Once the duplicate terms are removed, the unique terms are annotated and stored as 'vocabulary' that is used as a lookup to identify the terms for a new document.

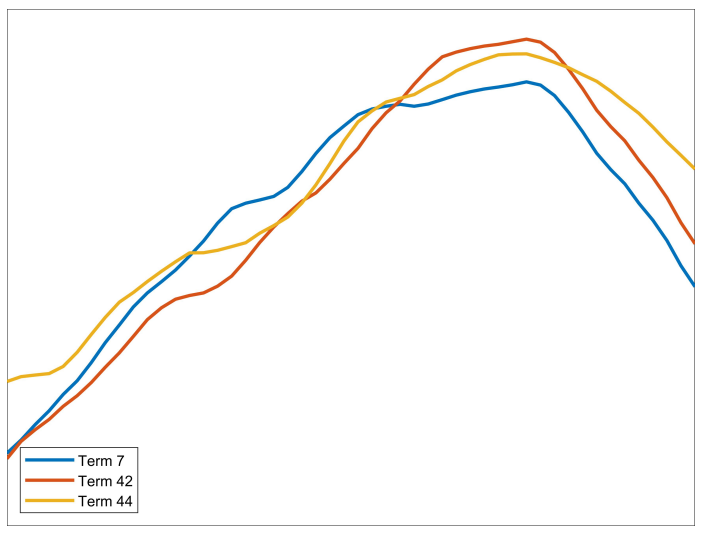

(a)

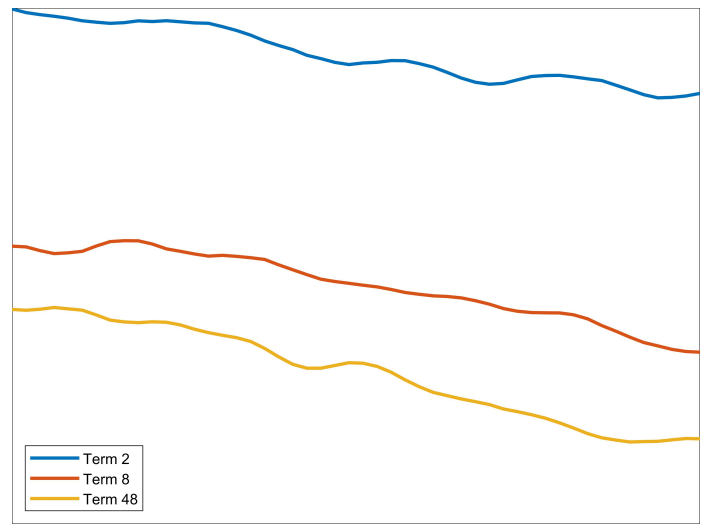

(b)

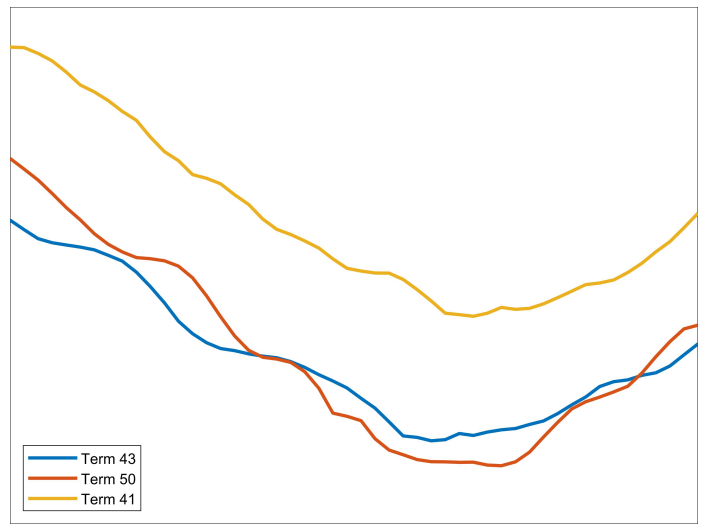

(c)

Fig. 4: Various terms from the magnetic field data have high similarity, (a) terms 7, 42 and 44, (b) terms 2, 8, and 38, (c) terms 43, 50 and 41.

\subsection{Localization Process}

The localization process involves two phases: the training phase and the testing phase. The former involves data collection, denoising, and transforming into terms and finding TF while the latter uses the trained model and the localization scheme to perform localization. Details of each of these phases are discussed separately. 


\subsubsection{Training Phase}

The training phase involves the transformation of the magnetic field data into terms and extraction of TF for ground truth positions. The TF is calculated using Equation 1, while Table 2 shows a specimen of TF vector. Each TF is formed using a data length of five seconds, covering approximately a distance of 4.5 to 5.0 $\mathrm{m}$. The use of five seconds data is based on the findings of the experiment where the impact of various lengths of data on the localization accuracy is investigated. Experiments show that a minimum of five seconds of data is necessary to achieve the desired positioning accuracy for indoors. Of course, a less amount of data can be used for positioning, however, it will degrade the positioning accuracy. Similarly, using a large amount of data would estimate a position with higher accuracy but increase the processing time latency. After the TF vector for each ground truth location is prepared, it is used to train two neural networks. This study uses long short term memory and gated recurrent unit networks in this regard.

The sequential relationship between the words plays an important role in text analysis. Recurrent neural network (RNN) is proposed in [30] for text sequence data. RNN has input, hidden and output layer like DNN does. However, it aggregates the input and hidden layer at time $t$ to make a new layer to calculate the hidden layer and its loop structure allows information to be persistent. In practical matters, RNN suffers from history information loss and gradient attenuation during training. Long short term memory (LSTM) is proposed to overcome the limitations of RNN and currently one of the most widely used ones for text analysis. RNN node is replaced with an LSTM cell that saves the text information history. Input, forget and output gates placed in LSTM cell helps in reading, saving, and updating history information [31].

The gated recurrent unit (GRU) proposed in [32] is another variant of RNN that uses each recurrent unit for capturing dependencies related to time scales. Analogous to LSTM, GRU is comprised of various gating units. These units are used to regulate information flow within the units. The total number of parameters in GRU is increased by 3 -fold as compared to simple RNN. It is observed in various studies, like in $[33,34]$ that the performance of GRU is comparable or leading to that of LSTM in many cases. These networks are trained using the architecture and parameters given in Table 4. Dropout layers are added to avoid model overfitting. Training data is split into training and validation with a ratio of 7 to 3 .
Table 4: Architecture and parameters used for LSTM and GRU for training and validation.

\begin{tabular}{|c|c|}
\hline Model & Parameters \\
\hline LSTM & $\begin{array}{l}\text { sequence input layer, LSTM layer (100), fully } \\
\text { connected layer (180) } \\
\text { fully connected layer (90), fully connected layer, } \\
\text { softmax layer }\end{array}$ \\
\hline GRU & $\begin{array}{l}\text { Optimizer='Adam', Epochs=2000, Learning } \\
\text { rate }=1 \mathrm{e}^{-4} \\
\text { sequence input layer, GRU layer (100), fully } \\
\text { connected layer (180), dropout layer }(0.5) \\
\text { fully connected layer (90), dropout layer }(0.5) \text {, } \\
\text { fully connected layer, softmax layer } \\
\text { Optimizer='Adam', Epochs=2000, Learning } \\
\text { rate }=1 \mathrm{e}^{-4}\end{array}$ \\
\hline
\end{tabular}

\subsubsection{Localization/Testing Phase}

The localization phase includes three sub-phases: transforming the magnetic field data into terms, searching the terms in the vocabulary, and making the TF vector for a particular location. Transformation of the magnetic field data is done using Algorithm 1 (lines 1-4). Once the term $t$ is defined, we need to search it in the vocabulary $V$ to identify it for counting its frequency. Lines 6-13 of Algorithm 1 are used for this purpose along with the criteria defined in Equation 5. The extracted TF vector is then used for prediction using the trained LSTM and GRU networks. Figure 5 shows the methodology adopted for the localization process.

Instead of using one neural network, this study utilizes two neural networks known for their good performance for text analysis and incorporates their predictions using a voting scheme to estimate the current location of the user. The preference of more than one network lies in the empirical evidence that prediction from one neural network occasionally leads to location prediction, far away from the original location. This leads to high maximum errors, as well as, affects the mean error and standard deviation of the localization algorithm. Voting more than one neural network helps to overcome this limitation and improves the performance of the localization approach. Location estimation is done using Algorithm 2.

The TF vector extracted from the magnetic field data for a location is the input for Algorithm 2, along with the trained LSTM and GRU networks. The output of the algorithm is the user's estimated current location.

Lines 1-2: of Algorithm 2 use the TF vector with the trained networks to predict the user's location. However, instead of taking a single prediction from these networks, this study considers top $k$ predictions along 


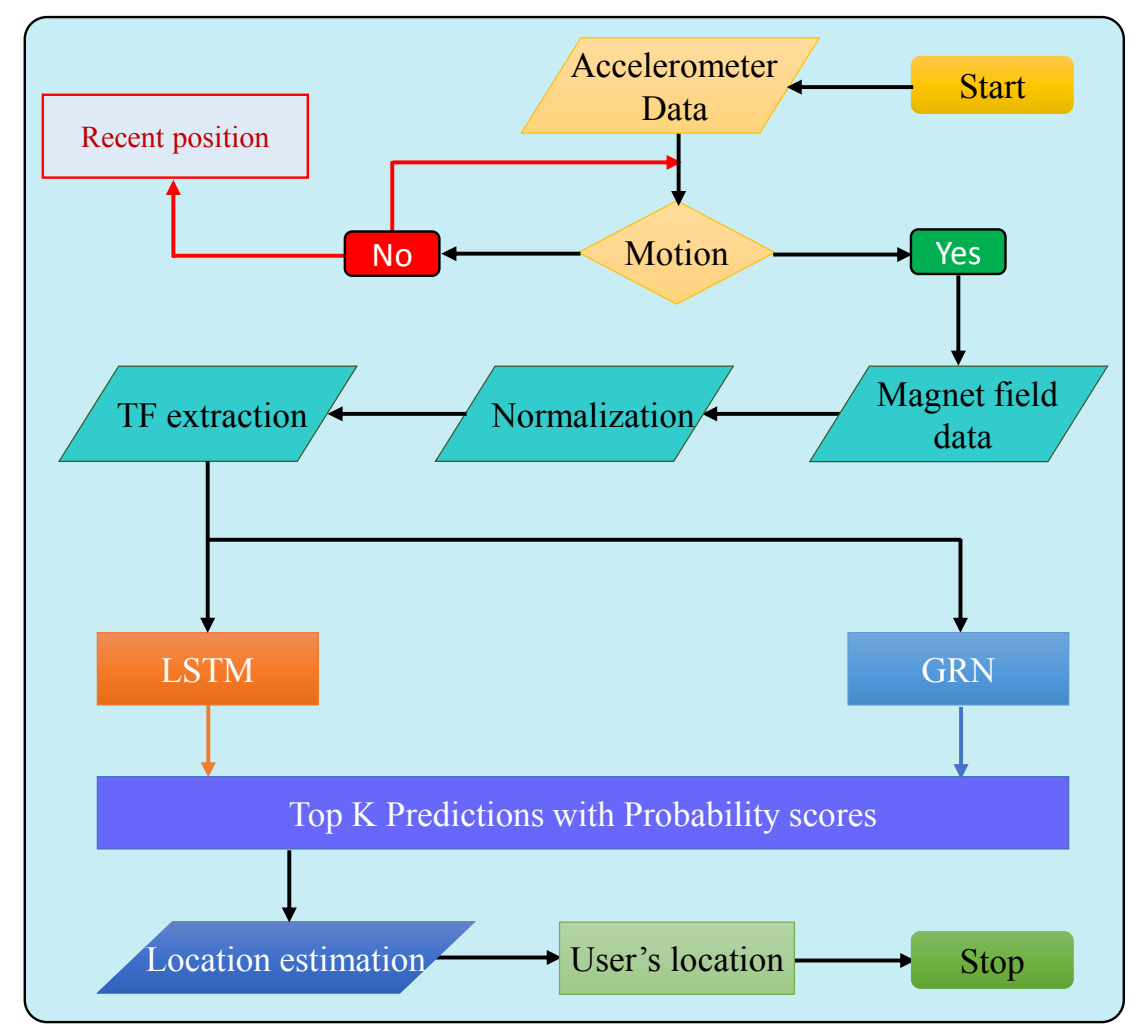

Fig. 5: The adopted methodology to estimate the location of the user.

with their prediction weights. The $k$ is based on the empirical findings of our exper iments and its value is set to 3 . On the other hand, prediction weights are the prediction scores for each prediction from the trained networks. We consider the weights because it gives the prediction confidence. For example, we found that if the prediction weight is high like 0.8 or higher, it is highly probable that it is the original location or very close to the original location of the user. On the contrary, the predictions may often carry smaller weights like 0.2 or lower which increases the uncertainty to predict the user's location. A reasonable solution for such scenarios would be to estimate the location based on the predictions from multiple neural networks, and that is exactly what we do.

Lines 3-4: finds unique positions from LSTM predictions $l_{\text {stm }}$ and GRU predictions gru $_{p}$ because often LSTM and GRU trained networks have one or more common predictions among $k$ taken predictions; the weights, at the same time, may not be the same. The weights $W$ for unique predictions $P$ should also be taken that are used to break the tie if more than one predictions have a similar occurrence.

Lines 5-8: are executed for each element of the unique predictions set $P$ to count the total occurrence of each prediction from $l_{s t m}$ and $g r u_{p}$. So, $L_{c}$ rep-

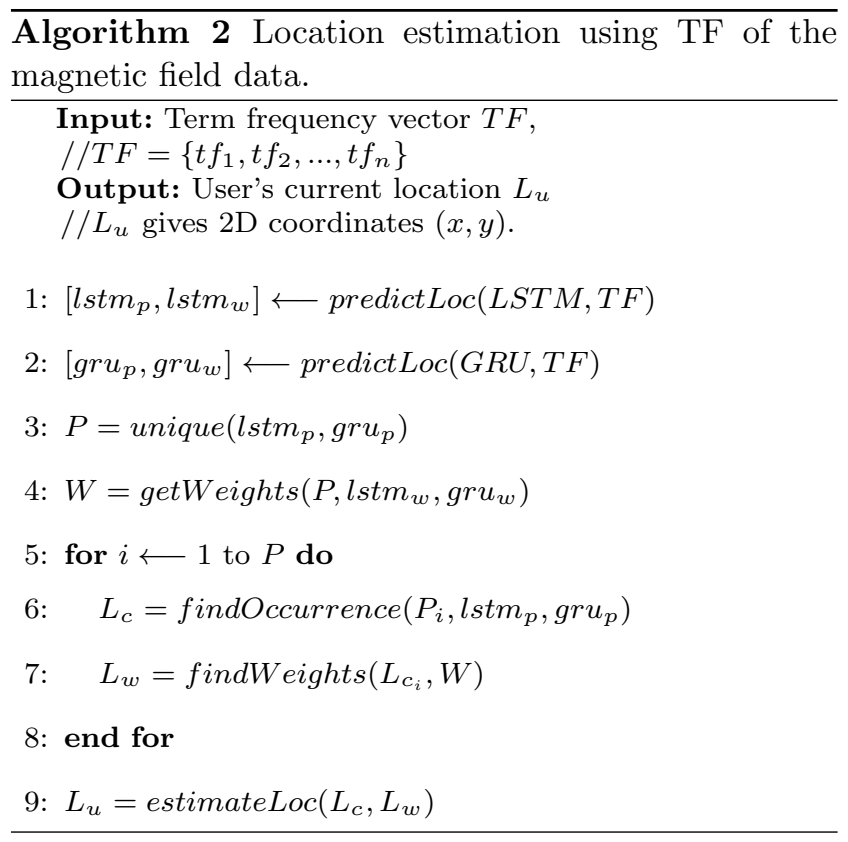

resents location candidates and is a $2 \times n$ matrix with columns as predictions and their occurrence while rows are $n$ unique predictions. Besides, $L_{w}$ is maintained to store the summed weight for predictions with higher than one occurrence. 
Lines 9 estimates the current location of the user. The following criteria are used for location estimation. First, find the location candidates whose occurrence is equal to 2 , i.e. the same prediction from both the neural networks.

$O=\operatorname{find}\left(L_{c}==2\right)$

Equation 6 counts the occurrence of each location candidate. The candidates with higher occurrences have a higher probability to be the user's current location. Location estimation is then made with

$$
\begin{cases}\text { length }(O)>1 & L_{u}=\operatorname{mean}(O) \\ \text { else } & L_{u}=O\end{cases}
$$

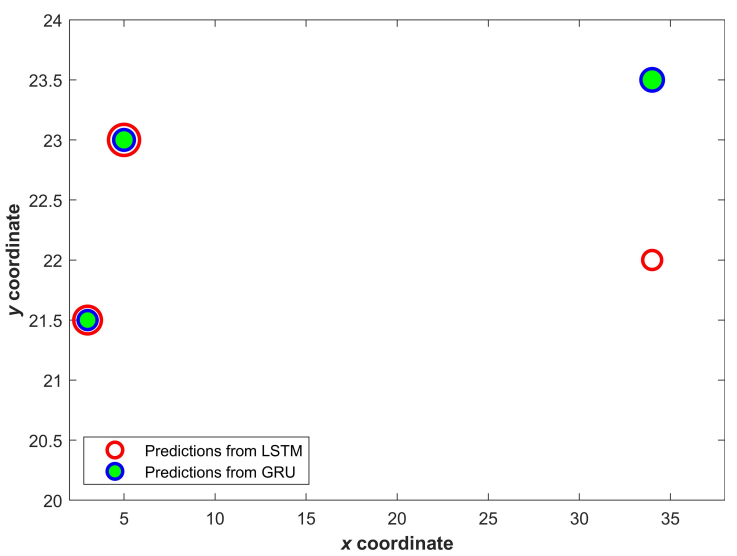

(a)

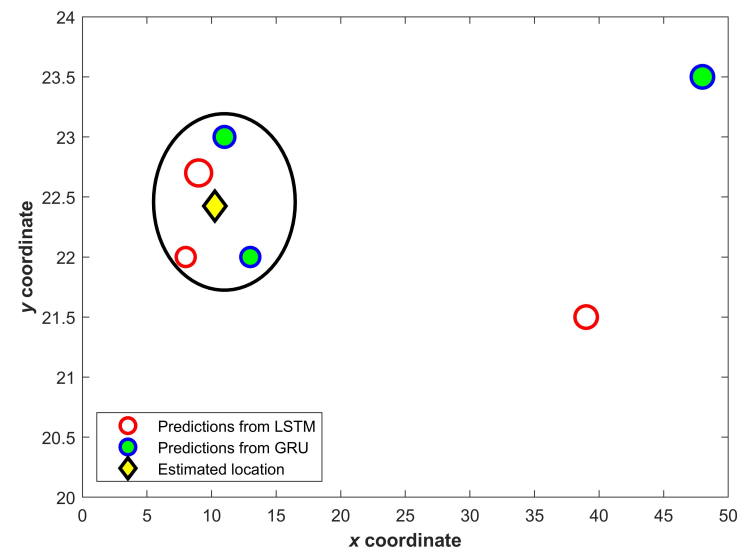

(b)

Fig. 6: Location estimation candidates and estimation process. The size of the circle indicate prediction score from LSTM and GRU, (a) scenario with two common predictions between SLTM and GRU, (b) scenario with no common prediction.
Predominantly, only one among $k$ considered predictions is common between $l_{s t m_{p}}$ and $g r u_{p}$, yet, more than such instances are also possible. In such scenarios, the mean of these instances serves as the estimated location. Figure 6(a) shows one such scenario where two predicted locations are common. Alternatively, if there is only one prediction that is common between lstm $_{p}$ and gru $_{p}$, it is the user's location.

The third possible scenario for location estimation is shown in Figure $6(\mathrm{~b})$ where each $k$ selected prediction from LSTM and GRU is unique. In that case the spatial proximity of $L_{c}$ to remove outlier. The elements in $L_{c}$ that are spatially close are considered for location estimation. The centroid of the selected candidates is the user's estimated location $L_{u}$ as shown in Figure 6(b).

\section{Results and Discussions}

The experiment setup and results are separately described in this section.

\subsection{Experiment Setup}

Experiments are carried out in a building of an area of $92 \times 36 \mathrm{~m}^{2}$. The path used to evaluate the performance of the proposed approach is shown in Figure 7. Three smartphones are used to analyze the results including LG G7, Samsung Galaxy S8, and LG Q6. Table 5 shows the parameter for conducted experiments.

Table 5: Experiment set up parameters.

\begin{tabular}{ll}
\hline Parameter & Description \\
\hline Smartphones used & LG G7, Galaxy S8, LG Q6 \\
Experiment area & $92 \times 36 \mathrm{~m}^{2}$ \\
Training data & Galaxy S8 - 12350 \\
& LG G6 - 6950 \\
Testing data & Galaxy S8 -6800 \\
& LG Q6 - 6850 \\
\hline
\end{tabular}

The smartphones used for the experiment have an embedded magnetic sensor that is used to collect the magnetic field data. The data from the Galaxy S8 is used for training while testing is performed with all the smartphones. The details of the specification of the smartphone magnetic sensors for all the smartphones are given in Table 6 . 


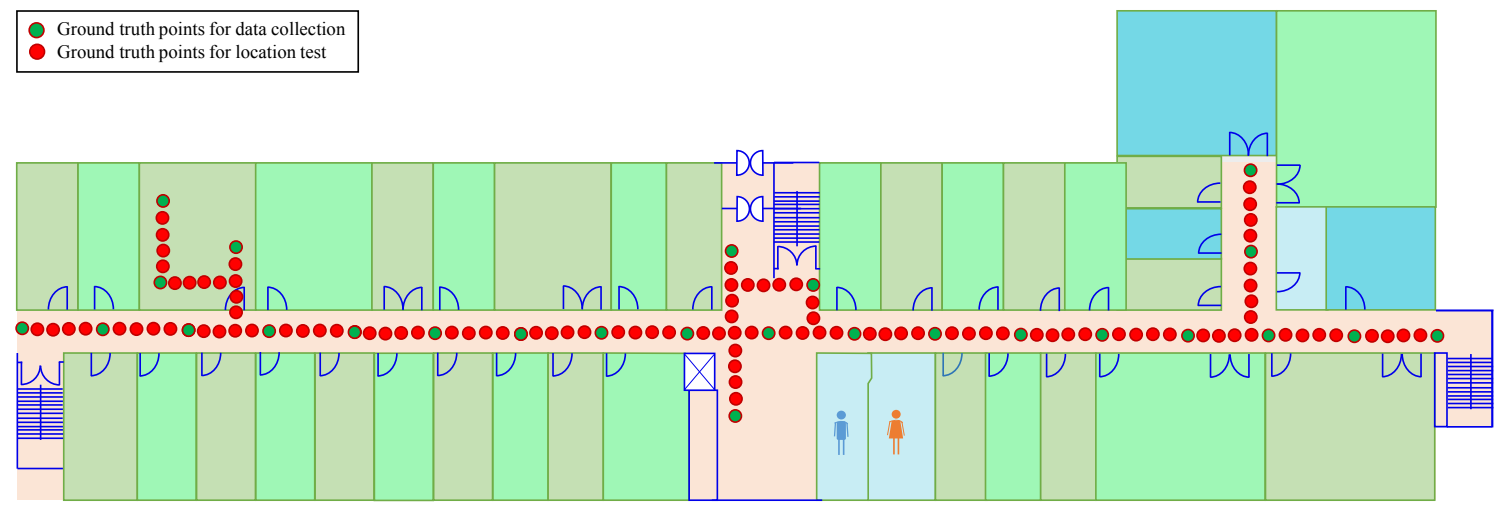

Fig. 7: The path used for experiments. Points on the map are separated by $1 \mathrm{~m}$ distance.

Table 6: List of sensors and their specifications used in the experiments

\begin{tabular}{|c|c|}
\hline Smartphone & Specification \\
\hline SM-G950N Galaxy S8 & $\begin{array}{l}\text { Octa-core, Adreno } 540 \text { GPU, } \\
\text { Android } 7.0 \text { (Nougat), } 4 \text { GB } \\
\text { RAM }\end{array}$ \\
\hline Magnetometer (AK09916C) & $\begin{array}{l}\text { 3-axis, } 16 \text {-bit, sensitivity } 0.15 \\
\mathrm{mT} / \mathrm{LSB} \text {, temperature }-30 \\
\text { to }+85^{\circ} \mathrm{C}, 6.0 \mathrm{~mA}[35]\end{array}$ \\
\hline LGM-G600L LG G6 & $\begin{array}{l}\text { Quad-core, Adreno } 530 \\
\text { GPU, Android } 7.0 \text { (Nougat), } \\
4 \text { GB RAM }\end{array}$ \\
\hline Magnetometer (AK09915C) & $\begin{array}{l}\text { 3-axis, } 16 \text {-bit, sensitivity } 0.15 \\
\mathrm{mT} / \mathrm{LSB} \text {, temperature }-30 \\
\text { to }+85^{\circ} \mathrm{C}, 6.0 \mathrm{~mA}[36]\end{array}$ \\
\hline LGM-X6OOS LG Q6 & $\begin{array}{l}\text { Octa-core, Adreno 505 GPU, } \\
\text { Android 7.1.1 (Nougat), } 3 \\
\text { GB RAM }\end{array}$ \\
\hline Magnetometer (AK09918C) & $\begin{array}{l}\text { 3-axis, } 16 \text {-bit, sensitivity } 0.15 \\
\mathrm{mT} / \mathrm{LSB} \text {, temperature }-30 \\
\text { to }+85^{\circ} \mathrm{C}, 1.1 \mathrm{~mA}[37]\end{array}$ \\
\hline
\end{tabular}

\subsection{Experiment Results}

Various experiments are performed for the current study to analyze the results of the proposed approach with the perspective of studying the impact of smartphone heterogeneity on the localization accuracy using the TF paradigm with the magnetic field data. Figure 8 shows the cumulative distributive function (CDF) graph for the localization accuracy with LG G6, Galaxy S8, and LG Q6. Results demonstrate that the localization using the proposed paradigm is similar if not identical using three different smartphones. The performance of Galaxy S8 is slightly better than those of the other two smartphones and the presumptive reason for this may be the use of S8 data for training. The localization performance of LG G6 and LG Q6 is almost identical while the maximum error for LG G6 is marginally high than those of S8 and Q6. Results for mean, maximum, and $50 \%$ error, etc. are shown in Table 7.

Table 7: Results details for the proposed approach using G6, S8 and Q6

\begin{tabular}{l|cccccc}
\hline \multirow{2}{*}{ Device } & \multicolumn{6}{|c}{ Distance error (m) } \\
\cline { 2 - 7 } & Mean & Med. & Max & S. D. & $\mathbf{5 0 \%}$ & $\mathbf{7 5 \%}$ \\
\hline LG G6 & 2.68 & 2.53 & 8.46 & 1.81 & 2.52 & 3.90 \\
Galaxy S8 & 2.56 & 2.27 & 8.45 & 1.81 & 2.26 & 3.62 \\
LG Q6 & 2.65 & 2.45 & 7.82 & 1.78 & 2.44 & 3.91 \\
\hline
\end{tabular}

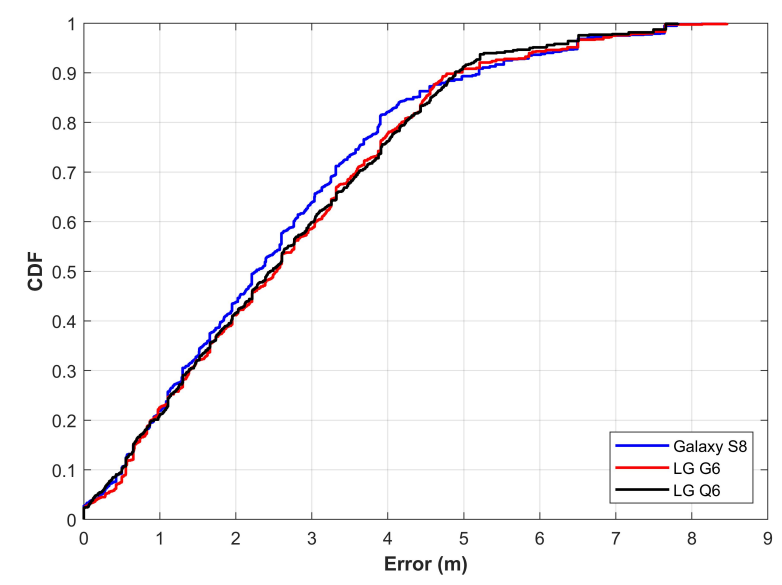

Fig. 8: CDF graph with the proposed approach for various smartphones used for experiments

Results shown in Table 7 indicate that the proposed approach can successfully alleviate the impact of smartphone heterogeneity on the localization accuracy when the magnetic field data is used for localization. The values for mean, median, $50 \%$, and $75 \%$ error are marginally different for the smartphones used for 
the experiments. Moreover, the localization accuracy is good and the user can be located within $2.54 \mathrm{~m}$ at $50 \%$, without regard to the smartphone used for localization. Similarly, $75 \%$ error is $3.90 \mathrm{~m}, 3.62 \mathrm{~m}$, and $3.91 \mathrm{~m}$ for LG G6, Galaxy S8, and LG Q6, respectively.

\subsection{Performance Comparison with Localization}

Approaches

Various approaches based on the magnetic field data are considered to analyze the performance of the proposed approach with two perspectives: capability of an approach to mitigate the impact of device heterogeneity and average localization accuracy. Figure 9 shows the localization performance of considered approaches including mPILOT [20], DeepLocate [22], and GUIDE [9] with different smartphones. It should be noted that the impact of using divergent smartphones for MFP approaches is more mitigative in the proposed approach shown in Figure 9(d). Other approaches, although, reduces this impact, yet, show large differences in perfor-

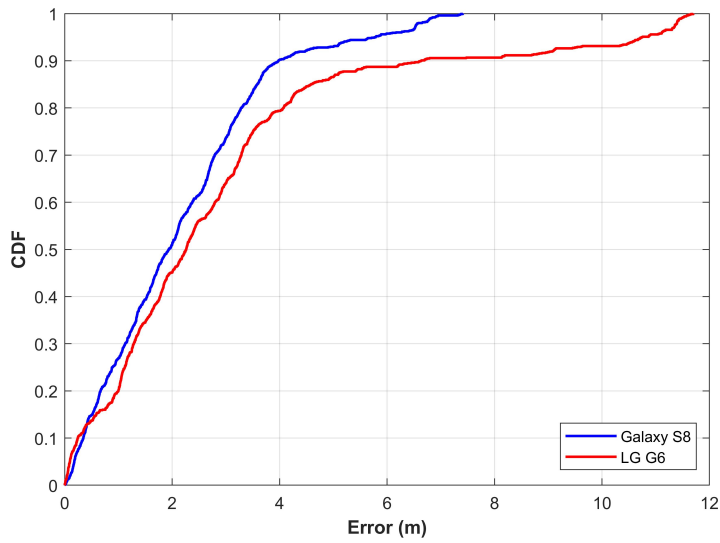

(a)

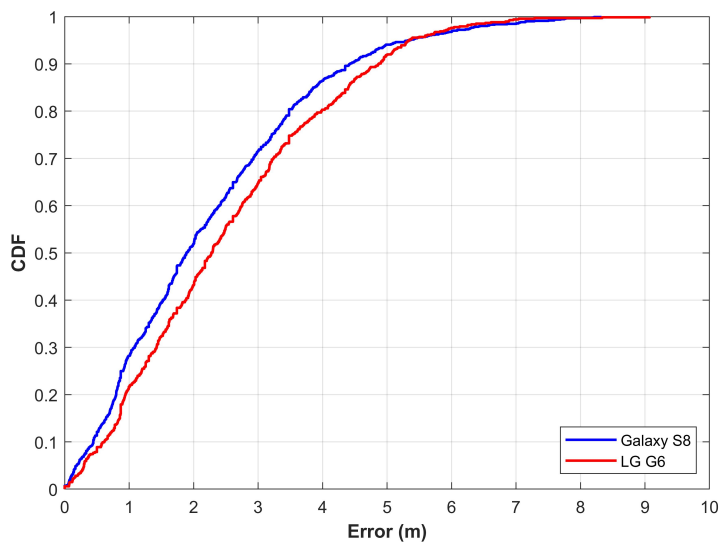

(c) mance with different smartphones like Figure 9 (a) and Figure 9(b). A detailed comparison in terms of error difference for mean, $50 \%$, and maximum errors, using the considered approaches would be more interesting, as shown in Table 8.

Table 8: Comparative results showing impact of various smartphonees on the localization accuracy using the magnetic field data.

\begin{tabular}{l|ccc}
\hline \multirow{2}{*}{ Approach } & \multicolumn{3}{|c}{ Error difference (m) } \\
\cline { 2 - 4 } & Mean & $\mathbf{7 5 \%}$ & Maximum \\
\hline mPILOT & 1.38 & 1.74 & 4.60 \\
GUIDE & 0.87 & 0.89 & 1.98 \\
DeepLocate & 0.53 & 0.34 & 2.12 \\
Propsed & 0.12 & 0.28 & 0.02 \\
\hline
\end{tabular}

The values for mean, $75 \%$, and maximum error in Table 8 show the absolute difference between Galaxy S8 and LG G6 errors with approaches taken for compari-

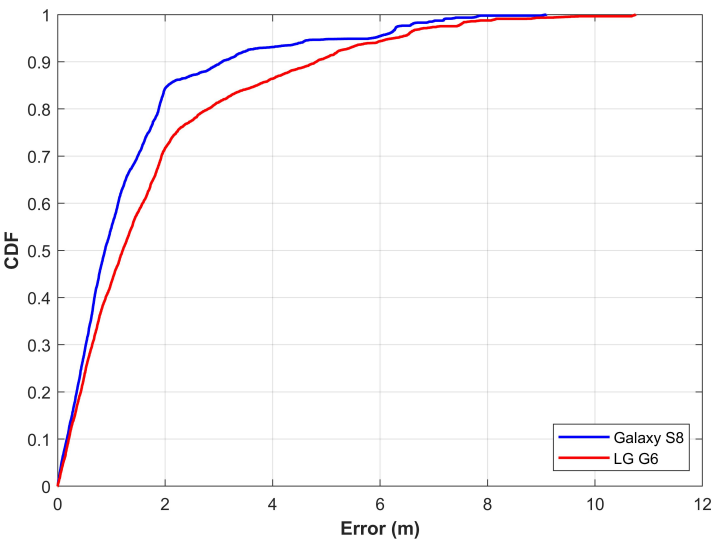

(b)

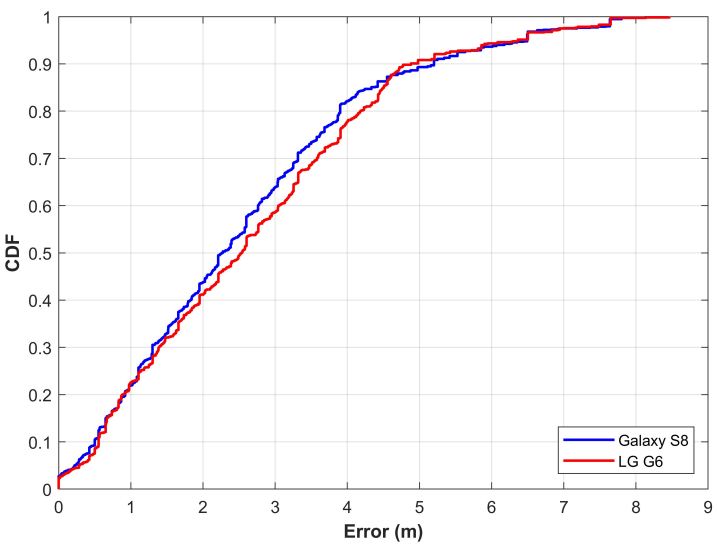

(d)

Fig. 9: Comparative CDF graphs of the proposed approach with other magnetic field based localization approaches with Galaxy S8 and LG G6, (a) mPILOT, (b) GUIDE, (c) DeepLocate, and (d) proposed approach. 
son. The given values do not represent the localization error, instead, it is the difference in the performance of various smartphones. The main objective of the proposed approach is to reduce the difference in positioning error when various smartphones are used for positioning. Results show that when the proposed approach has been used, the difference in the positioning error is lower. The difference in localization of various smartphone is low with the proposed approach than other approaches. Although the magnetic field data varies with different smartphones, the transformation of the data into terms helps to reduce the variability. Furthermore, the normalization process preserves the patterns of the magnetic field data which plays an important role to alleviate smartphone heterogeneity to improve the performance of the proposed approach.

Results demonstrate that there is a marginal difference in the performance of three different smartphones with the proposed approach than those of other magnetic field-based localization approaches. It shows the dominant performance of the proposed approach. Performance analysis is also done for the localization performance of the proposed approach with the selected approaches. Figure 10 shows the CDF graph for localization accuracy with all the approaches. Apparently, the localization accuracy of the proposed approach is slightly lower than those of other approaches. It is important to point out that our chief objective is the mitigation of device dependency for MFP approaches. However, further analysis for localization accuracy is made in Table 9 that revels that the localization performance of the proposed approach is better than other approaches as it uses smaller magnetic field data in comparison. Presumably, increasing the length of terms (magnetic field data) would yield higher accuracy than those of other approaches.

Table 9 show the details for mean, $50 \%, 75 \%$, and maximum error with the proposed approach and other similar approaches. Localization results for mPILOT are using $14 \mathrm{~s}$ of data while that of DeepLocate with $6 \mathrm{~s}$. In contrast, the proposed approach uses only $5 \mathrm{~s}$ data and localization performance is better than that of mPILOT. The mean and $75 \%$ error is comparable with DeepLocate while the maximum error is lower than that of DeepLocate. In light of the discussed results, we can say that the proposed TF paradigm is very effective in reducing the smartphone dependency that the existing MFP approaches have.

It is also noteworthy to point out that the localization accuracy comparison is not possible with [23]. Although, this work is similar to ours, yet it does not

\footnotetext{
1 Indicates the length of the data used to calculate the location.
}

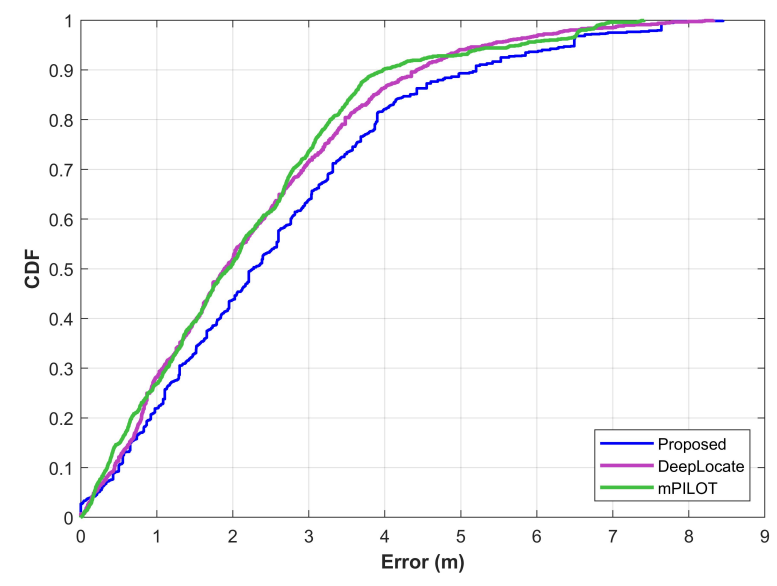

Fig. 10: CDF graph for the proposed approach and mPILOT and DeepLocate.

Table 9: Detailed statistics for performance comparison of the proposed approaches with other approaches.

\begin{tabular}{l|c|ccc|l}
\hline \multirow{2}{*}{ Approach } & \multirow{2}{*}{ Device } & \multicolumn{3}{|c|}{ Error difference (m) } & \multirow{2}{*}{ Data $^{1}$} \\
\cline { 3 - 5 } & & Mean & $\mathbf{7 5 \%}$ & Max & \\
\hline \multirow{2}{*}{ mPILOT } & Galaxy S8 & 2.92 & 4.01 & 7.60 & \multirow{2}{*}{$14 \mathrm{~s}$} \\
& LG G6 & 4.30 & 5.75 & 11.85 & \\
\hline \multirow{2}{*}{ DeepLocate } & Galaxy S8 & 2.23 & 3.21 & 8.32 & \multirow{2}{*}{$6 \mathrm{~s}$} \\
& LG G6 & 2.52 & 3.55 & 10.44 & \\
\hline \multirow{2}{*}{ Proposed } & Galaxy S8 & 2.26 & 3.90 & 8.45 & \multirow{2}{*}{$5 \mathrm{~s}$} \\
& LG G6 & 2.68 & 3.91 & 8.46 & \\
\hline
\end{tabular}

measure the performance of the proposed approach in terms of localization error, rather, it evaluates the effectiveness of the BoW approach for its classification accuracy. The current study, at the same time, focuses on the localization accuracy in $\mathrm{m}$ to analyze the effectiveness of the TF paradigm for mitigating the influence of heterogeneous devices. Additionally, research [23] does not review the BoW approach with multiple smartphones and its results can not be generalized.

\section{Conclusions}

This study investigates the use of the magnetic field data in challenging GNSS environments like near and in buildings scenarios where the global positioning system can not provide a reliable position. Magnetic field-based indoor positioning and localization is an attractive solution, yet, its accuracy is severely affected when multifarious smartphones are used for positioning. Owing to the large variety of smartphones available today, devising an approach that can work seamlessly with various smartphones is of significant importance. This study presents the term frequency paradigm from the text analysis domain to solve this issue. The magnetic field 
data is transformed into terms and a combination of these terms represents a word (specific location in this case). Extracted TF vectors are used to train LSTM and GRU networks and their predictions are voted to estimate the current location of the user.

Experiments are carried out using three different smartphones including LG G6, Samsung Galaxy S8, and LG Q6. Experimental results demonstrate that the proposed approach helps to alleviate the impact of device dependency on magnetic field-based localization. Performance comparison with three state-of-the-art approaches indicates that the proposed approach is more influential in mitigating the device dependence than those of other techniques. In addition, mean, maximum, $50 \%$, and $75 \%$ error are low than other techniques even using a smaller amount of the magnetic field data. The approach can localize the user withing $2.52 \mathrm{~m}$, and 3.91 $\mathrm{m}$ at $50 \%$ and $75 \%$, regardless of the three smartphones used for localization. A natural extension to the current study would be to test more smartphones and increase the localization accuracy of the proposed approach.

Acknowledgements This work was supported by the MSIT (Ministry of Science, ICT), Korea, under the ITRC (Information Technology Research Center) support program (IITP2020-2016-0-00313) supervised by the IITP (Institute for Information \& communication Technology Promotion). This research was supported by Basic Science Research Program through the National Research Foundation of Korea (NRF) funded by the Ministry of Science, ICT and Future Planning (2017R1E1A1A01074345).

\section{Conflict of interest}

"The authors declare no conflict of interest. The funding agency had no role in the design of the study; in the collection, analyses, or interpretation of data; in the writing of the manuscript, or in the decision to publish the results".

\section{References}

1. Realini, E., Caldera, S., Pertusini, L., Sampietro, D.: Precise gnss positioning using smart devices. Sensors 17(10), 2434 (2017)

2. Karimi, H.A.: Advanced location-based technologies and services. CRC Press (2016)

3. Alarifi, A., Al-Salman, A., Alsaleh, M., Alnafessah, A., Al-Hadhrami, S., Al-Ammar, M.A., Al-Khalifa, H.S.: Ultra wideband indoor positioning technologies: Analysis and recent advances. Sensors 16(5), 707 (2016)

4. Cantón Paterna, V., Calveras Auge, A., Paradells Aspas, J., Perez Bullones, M.A.: A bluetooth low energy indoor positioning system with channel diversity, weighted trilateration and kalman filtering. Sensors 17(12), 2927 (2017)
5. Yao, C.Y., Hsia, W.C.: An indoor positioning system based on the dual-channel passive rfid technology. IEEE Sensors Journal 18(11), 4654-4663 (2018)

6. Ali, M.U., Hur, S., Park, S., Park, Y.: Harvesting indoor positioning accuracy by exploring multiple features from received signal strength vector. IEEE Access 7, 5211052121 (2019)

7. Zhuang, Y., Syed, Z., Li, Y., El-Sheimy, N.: Evaluation of two wifi positioning systems based on autonomous crowdsourcing of handheld devices for indoor navigation. IEEE Transactions on Mobile Computing 15(8), 1982 1995 (2015)

8. Ashraf, I., Hur, S., Park, Y.: Indoor positioning on disparate commercial smartphones using wi-fi access points coverage area. Sensors 19(19), 4351 (2019)

9. Ashraf, I., Hur, S., Shafiq, M., Kumari, S., Park, Y.: Guide: Smartphone sensors-based pedestrian indoor localization with heterogeneous devices. International Journal of Communication Systems 32(15), e4062 (2019)

10. Pasku, V., De Angelis, A., De Angelis, G., Arumugam, D.D., Dionigi, M., Carbone, P., Moschitta, A., Ricketts, D.S.: Magnetic field-based positioning systems. IEEE Communications Surveys \& Tutorials 19(3), 2003-2017 (2017)

11. Galván-Tejada, C.E., Carrasco-Jimenez, J.C., Brena, R.: Location identification using a magnetic-field-based fft signature. Procedia Computer Science 19, 533-539 (2013)

12. Gozick, B., Subbu, K.P., Dantu, R., Maeshiro, T.: Magnetic maps for indoor navigation. IEEE Transactions on Instrumentation and Measurement 60(12), 3883-3891 (2011)

13. Lee, N., Han, D.: Magnetic indoor positioning system using deep neural network. In: 2017 International Conference on Indoor Positioning and Indoor Navigation (IPIN), pp. 1-8. IEEE (2017)

14. Gu, F., Niu, J., Duan, L.: Waipo: A fusion-based collaborative indoor localization system on smartphones. IEEE/ACM Transactions on Networking 25(4), 22672280 (2017)

15. Lohan, E.S., Talvitie, J., e Silva, P.F., Nurminen, H., AliLöytty, S., Piché, R.: Received signal strength models for wlan and ble-based indoor positioning in multi-floor buildings. In: 2015 International Conference on Localization and GNSS (ICL-GNSS), pp. 1-6. IEEE (2015)

16. Huang, K., He, K., Du, X.: A hybrid method to improve the ble-based indoor positioning in a dense bluetooth environment. Sensors 19(2), 424 (2019)

17. Ma, Z., Poslad, S., Bigham, J., Zhang, X., Men, L.: A ble rssi ranking based indoor positioning system for generic smartphones. In: 2017 Wireless Telecommunications Symposium (WTS), pp. 1-8. IEEE (2017)

18. Liu, M., Chen, R., Li, D., Chen, Y., Guo, G., Cao, Z., Pan, Y.: Scene recognition for indoor localization using a multi-sensor fusion approach. Sensors 17(12), 2847 (2017)

19. Shu, Y., Bo, C., Shen, G., Zhao, C., Li, L., Zhao, F.: Magicol: Indoor localization using pervasive magnetic field and opportunistic wifi sensing. IEEE Journal on Selected Areas in Communications 33(7), 1443-1457 (2015)

20. Ashraf, I., Hur, S., Park, Y.: mpilot-magnetic field strength based pedestrian indoor localization. Sensors 18(7), 2283 (2018)

21. Subbu, K.P., Gozick, B., Dantu, R.: Locateme: Magneticfields-based indoor localization using smartphones. ACM Transactions on Intelligent Systems and Technology (TIST) 4(4), 1-27 (2013) 
22. Ashraf, I., Hur, S., Park, S., Park, Y.: Deeplocate: Smartphone based indoor localization with a deep neural network ensemble classifier. Sensors 20(1), 133 (2020)

23. Montoliu, R., Torres-Sospedra, J., Belmonte, O.: Magnetic field based indoor positioning using the bag of words paradigm. In: 2016 International Conference on Indoor Positioning and Indoor Navigation (IPIN), pp. 1-7. IEEE (2016)

24. Li, B., Gallagher, T., Dempster, A.G., Rizos, C.: How feasible is the use of magnetic field alone for indoor positioning? In: 2012 International Conference on Indoor Positioning and Indoor Navigation (IPIN), pp. 1-9. IEEE (2012)

25. Gunnarsdóttir, E.L.: The earth's magnetic field. Ph.D. thesis (2012)

26. Zhang, W., Yoshida, T., Tang, X.: A comparative study of tf* idf, lsi and multi-words for text classification. Expert Systems with Applications 38(3), 2758-2765 (2011)

27. Bai, Y., Yu, W., Xiao, T., Xu, C., Yang, K., Ma, W.Y., Zhao, T.: Bag-of-words based deep neural network for image retrieval. In: Proceedings of the 22nd ACM international conference on Multimedia, pp. 229-232 (2014)

28. Pennington, J., Socher, R., Manning, C.D.: Glove: Global vectors for word representation. In: Proceedings of the 2014 conference on empirical methods in natural language processing (EMNLP), pp. 1532-1543 (2014)

29. Mikolov, T., Yih, W.t., Zweig, G.: Linguistic regularities in continuous space word representations. In: Proceedings of the 2013 conference of the north american chapter of the association for computational linguistics: Human language technologies, pp. 746-751 (2013)

30. Hochreiter, S., Schmidhuber, J.: Long short-term memory. Neural computation 9(8), 1735-1780 (1997)

31. Graves, A.: Supervised sequence labelling with recurrent neural networks [ph. d. dissertation]. Technical University of Munich, Germany (2008)

32. Cho, K., Van Merriënboer, B., Bahdanau, D., Bengio, Y.: On the properties of neural machine translation: Encoder-decoder approaches. arXiv preprint arXiv:1409.1259 (2014)

33. Chung, J., Gulcehre, C., Cho, K., Bengio, Y.: Empirical evaluation of gated recurrent neural networks on sequence modeling. arXiv preprint arXiv:1412.3555 (2014)

34. Yao, K., Cohn, T., Vylomova, K., Duh, K., Dyer, C.: Depth-gated recurrent neural networks. arXiv preprint arXiv:1508.03790 9 (2015)

35. Asahi Kasei Microdevices Corporation: AK09916C. https://www.akm.com/akm/en/file/datasheet/ AK09916C.pdf. Online: accessed 20 April 2020

36. Asahi Kasei Microdevices Corporation: AK09915C. https://www.akm.com/global/en/products/electroniccompass/ak09915c/. Online: accessed 19 April 2020

37. Asahi Kasei Microdevices Corporation: AK09918C. https://www.akm.com/global/en/products/electroniccompass/ak09918c/. Online: accessed 19 April 2020 